\title{
28
}

\section{Assessing the quality of specification-based testing}

\author{
S.P. Allen and M.R. Woodward \\ University of Liverpool \\ Department of Computer Science, P.O. Box 147, Liverpool, L69 3BX, \\ U.K. Tel: +441517943670 Fax: +441517943715 email: \\ $\{$ spa,mrw\}@csc.liv.ac.uk
}

\begin{abstract}
This paper reports an experimental investigation into the quality of sets of test expressions automatically generated from algebraic specifications. Faults were introduced into implementations in $\mathrm{C}$ and Fortran equivalent to the chosen specifications, manually in the case of the $\mathrm{C}$ versions, and using the Mothra mutation system in the case of the Fortran versions. The algebraic specifications themselves were also subject to mutation analysis. A number of test generation strategies were considered, in order to circumvent the problem of handling prohibitively large numbers of test expressions. Results show that certain test sets detected the majority of faults and appear to perform better than random test sets of comparable sizes.
\end{abstract}

Keywords

Specification-based testing, error-seeding, mutation, test experiments

\section{INTRODUCTION}

The bewildering variety of different testing methodologies means that software testers often rely on experience and intuition to carry out their work. With more and more programs being specified by using a formal method, it appears feasible to use these formal specifications to derive appropriate test cases. A formal specification is a description of a program or module; it only describes what has to be done to produce the required result, not how it is to be done. At present most testing techniques rely on the execution of the program to give an indication of what is happening. These methods usually require an inspection of the program to select test data that is likely to expose faults. It is felt that a testing methodology based upon the formal description of a program will provide a systematic way of generating tests that will expose a substantial number of faults. 
There are many different types of formal specification notation. The model-based sequential notations of Z and VDM (Vienna Development Method) are among the most popular. Property-based sequential notations using an algebraic style are another popular approach. The specific focus of interest here is the algebraic notation OBJ, for two reasons (i) OBJ is executable and (ii) a tool is available that automatically generates test expressions from an OBJ specification. It is hoped that full advantage can be taken of these two features in this research.

The earliest work done in this field concentrated on test expression generation from algebraic specifications of abstract data types (ADTs) because these are the most understood and most mathematical aspects of a system's design. Perhaps the most widely known work in this area is the DAISTS system developed by Gannon et al (1981). Gerrard et al (1990) have done research into design time testing using algebraic specifications; they believe one of the best ways of ensuring correct specifications is being able to add unvalidated objects to correct environments. This way if a failure occurs it is straightforward to determine where the fault is located. The ability to execute the specification is an advantage in this case. Many ideas have been suggested for generating test data from specifications. Stocks and Carrington (1993) have presented a framework for generating general descriptions of test sets by specifying what constraints should be applied to the test data. The framework was applied to Z specifications. Hall (1988) has discussed the problems of automating the generation of test cases. Jalote (1989) suggested that meaningful test sets can be generated by just considering the syntactic aspect of the formal specification. Doong and Frankl (1991) though, indicate through their research, that it is very important to consider the other aspect of a specification, that is, the semantic part, as different initial values of parameters can lead to many different states. Building on the work of Bougé et al (1986), Bernot et al (1991) have conducted extensive research into generating test data from algebraic specifications by translating them into Horn clauses. A tool implemented using Prolog simulates equational resolution and provides control mechanisms for generating the test data. Very little research has been conducted on assessing the quality of test data automatically generated from specifications in terms of its effect on equivalent implementations.

This paper considers the feasibility of automatically generating test expressions from a formal specification and reports on experiments aimed at determining how good these expressions are at exposing faults in implementations. Two medium-sized OBJ specifications were selected and equivalent implementations in $\mathrm{C}$ and Fortran were developed. Faults were seeded into the $\mathrm{C}$ programs and mutants were generated of the Fortran programs and the OBJ specifications themselves. These faulty versions were used to assess the quality of test expressions generated from the OBJ specifications. In section 2 an overview of OBJ is given, as is a brief description of some of the tools that were used to perform this study. Section 3 describes in more detail the experiments carried out. Section 4 outlines the results that were achieved and some of the interesting points raised by this work. Finally section 5 summarises this work.

\section{OBJ AND TOOLS AVAILABLE}

Given below are brief descriptions of some of the tools that were used in assessing the quality of automatically generated test expressions. 


\subsection{OBJ}

OBJ is an algebraic specification language a full introduction to which can be found in the work of Goguen and Tardo (1979). The version used in this study was developed at UMIST (Coleman et al, 1987). At its simplest level an OBJ specification consists of a collection of objects; an object starts with the keyword OBJ and ends with JBO. An object can consist of up to four sections: SORTS, OPS, VARS and EQNS. The SORTS section describes the abstract data type that will be used in the object. The OPS section describes the operators required in terms of the range and domain of each operator. The VARS section is for declaring the symbols needed for the EQNS section. This last section describes the behaviour of operators by means of equations which indicate their relationships with one another. An equation may hold only if a certain condition is true; this may be expressed by means of an optional if clause. The only built in sorts are NAT, the natural numbers, with the usual arithmetic operations being defined, i.e. +, /, -, * etc and BOOL, the booleans $\mathrm{T}$ and $\mathrm{F}$ with predefined logical operators and, or and not.

OBJ specifications unlike most other types of formal notation are executable. This is achieved by term rewriting. When a test expression is presented to the OBJ interpreter if the left hand side of an equation matches with elements in the expression, it is replaced by the corresponding right hand side of the equation. This continues until it is not possible to repeat the matching process and the result is returned. An example in OBJ below shows part of a specification, given by Gerrard et al (1990), which defines a List ADT.

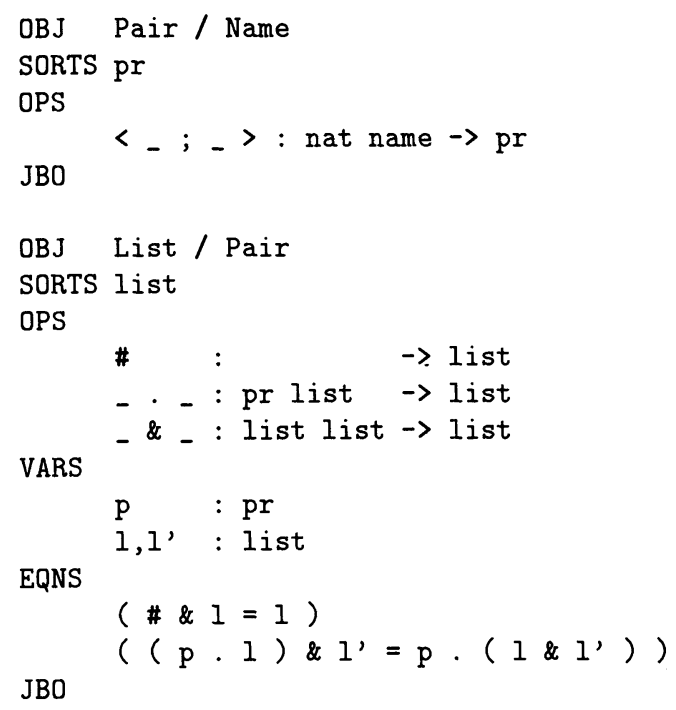

If $a a, b b$ and $c c$ are valid names then the following expression creates a list containing three elements:

$\langle 0 ; \mathrm{aa}\rangle .\langle 1 ; \mathrm{bb}\rangle .\langle 2 ; \mathrm{cc}\rangle$.\# 
Adding a list of one element to another (empty) list, as in the following expression:

$\# \&<0 ;$ aa $>$. \#

will produce, using the first equation for term rewriting, the result:

$\langle 0$; aa $>$.\#

\subsection{Test expression generator OBJTEST}

OBJTEST (Woodward, 1993) automatically generates test expressions from an OBJ specification. Test expressions are generated by taking each operator in turn and substituting for the arguments in the domain each operator whose range is of the appropriate sort. Testing the List ADT described above, OBJTEST will produce, in addition to others, expressions like:

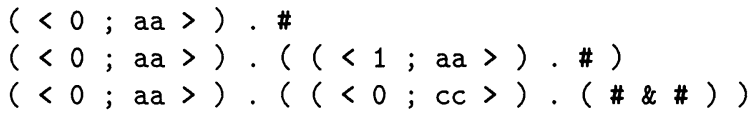

OBJTEST has many options that can be used to help generate test expressions. The most important of these are:

- The ability to select specific modules from a complete specification to test.

- The ability to specify the nesting depth of test expressions. Here expressions are generated as described above and repeated until the depth of nesting as specified by the user has been reached.

- The ability to de-select a constant from being used to generate test expressions. This is useful because many test expressions are seen to be equivalent. For example, if for the List ADT described above, many more numbers and names were defined then all combinations would be produced using the additional values but many expressions would be essentially the same. These can be considered as symmetrical expressions.

Additionally OBJTEST has another facility, the ability to generate mutants from an OBJ specification. This is achieved by introducing minor modifications to the specification. Again it is possible to specify which modules are to be tested and what type of mutant is to be generated. The version of OBJTEST that was being used generated four classes of mutant. They were:

- Similar variable replacement. A variable is replaced with a different variable of the same type.

- Operator attribute alteration. If an operator is for example, associative and commutative, one mutation would be to remove the associativity.

- Equation removal. Each operator is defined normally by one or more equations; with this mutation each equation is removed one at a time.

- Similar operator replacement. An operator is replaced with a different operator but having the same domain and range. 
An example of a mutant generated from the List ADT would be to replace 1 by l' on the right hand side of the second equation resulting in the following:

$$
\left((p \cdot I) \& I^{\prime}=p \cdot\left(I^{\prime} \& I^{\prime}\right)\right)
$$

Being executable, the mutant specifications can then be tested in an attempt to distinguish them from the original.

\subsection{Mothra}

Mothra (King and Offutt, 1991) is a mutation testing system for Fortran programs. It incorporates 22 mutation operators and the user can specify which of them are to be used. Mothra generates mutants of Fortran programs and by using test sets, supplied by the tester or automatically generated by Mothra, determines which mutants are killed by the test set.

When a test run has been completed Mothra generates comprehensive results of the mutant killing ability of the test set. It is up to the user to identify equivalent mutants, although recently some algorithms have been produced that attempt to identify equivalent mutants (Offutt and Craft, 1994).

\section{ASSESSING THE QUALITY OF AUTOMATICALLY GENERATED TEST EXPRESSIONS}

When designing experiments to assess the quality of automatically generated test expressions, the choice of subject programs and specifications is very important. Ideally both the program and specification should be available. Obtaining reasonable sized programs and their corresponding formal descriptions was discovered to be very difficult. For this experiment a student database system (Rolland, 1992) was used, given as a formal description in VDM and as an equivalent program in Modula-2. Also used was a nontrivial airport scheduling specification, given in OBJ by Goguen and Tardo (1979). The unavailability of large OBJ specifications made it necessary to translate the VDM specification of the student database into OBJ. It is acknowledged that this method is not ideal and was done to overcome the aforementioned problem. The equivalent implementation of the student database was translated into $\mathrm{C}$ and Fortran. The OBJ airport specification was used to develop implementations in $\mathrm{C}$ and Fortran.

\subsection{Subject programs}

Below is a brief description of the sample programs and specifications used.

\section{Student Database}

The student database is designed as a set of student details: name, class and the sex of each student. The OBJ specification is implemented using a Set ADT. The C and Fortran versions were implemented accordingly. There are seven operations that manipulate the database; they are: initialise, addmale, addfemale, deletemale, deletefemale, findmales and findfemales. Examples of typical operations allowed on the OBJ specification are: 
addmale (Fred, 0 , Null )

addmale (Fred , 0 , addmale (Fred, 1, Null U Null ) )

findmales (deletefemale (June, 1 , addmale (Fred, 0 , Null ) ) )

where Null is the empty set (or empty database) and $U$ is the set union operator.

Two values each were chosen for the name and class parameters. No restrictions were placed on the length of names or the size of the class. Fred and June were chosen as the names and 0 and 1 as the class numbers. Test expressions were generated using OBJTEST together with various constraints that it was believed would provide insight into the ability of test expressions generated from an algebraic specification to expose faults in implementations. These are described in section 4.

\section{Airport Specification}

The airport specification is a much larger specification than the student database. Essentially there are three modules that deal with different aspects of scheduling airplanes, with another module that brings the other three modules together. There are additional minor modules that check the validity of the flight numbers, plane numbers and time. Typical operations may be:

schedule ( Fl 1, LAX, 13 hrs 45, Pl 2, DC9, create )

This schedules flight 1 to go to LAX at 13:45 using plane 2 which is a DC9,

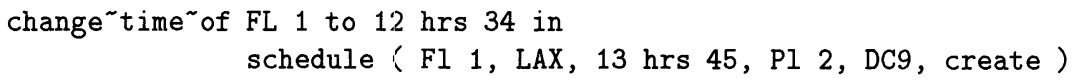

This changes the time of flight 1 to $12: 34$.

cancel Fl 5 in schedule ( Fl 1, LAX, 13 hrs 45, Pl 2, DC9, create)

This cancels flight 5 details from the airport schedule.

Three values each for the flight number, plane number and times were chosen and only one of the plane types and destinations available were used. Also four constants were added that represented one, two, three and four flight details. The constant representing four complete flight details was called TODAY and was an example given with the original specification. Values for the times, flight numbers and plane numbers included invalid values.

\subsection{Using test expressions generated from OBJ specification}

The test expressions generated from the OBJ specifications described previously, were used to test the $\mathrm{C}$ and Fortran implementations. The test expressions were read in and translated into a form that could be used to test the implementations. There was a direct relationship between the operators of the OBJ specifications and the procedures of the $\mathrm{C}$ and Fortran implementations. 
Table 1 Number and type of faults seeded into C programs

\begin{tabular}{llrr}
\hline Subject & Fault Category & Omission & Commission \\
\hline Student & Data Definition & 3 & 6 \\
Database & Data Handling & 3 & 4 \\
& Decision and processing & 5 & 0 \\
& Decision & 4 & 2 \\
Airport & Data Definition & 6 & 12 \\
Scheduler & Data Handling & 6 & 6 \\
& Decision and processing & 5 & 6 \\
& Decision & 9 & 1 \\
\hline
\end{tabular}

\subsection{Assessing the quality of test expressions}

To assess the quality of test expressions faults were seeded into the $\mathrm{C}$ programs. The intention was to make the type and number of faults as far as possible representative of the type and number of faults found in real world situations. To this end the seeded faults were modeled in type and frequency on the result of a study of industrial software by Ostrand and Weyuker (1984). This study classified faults in two ways. Faults could either be errors of omission, i.e., something was left out of the code causing it to function incorrectly, or errors of commission, i.e., something was added to the code to make the software function incorrectly. The fault detection ability of the test expressions was assessed by determining the ratio of the exposed faults to the number of seeded faults. To supplement this limited experiment, mutation analysis was also used. Using Mothra, mutants of the Fortran programs were generated and the quality of test expressions was assessed against these mutants. Additionally mutants of the original OBJ specification were generated and the mutant killing ability of the test expressions on the specification itself was assessed. It is believed that by determining how many mutants are killed by a test set, that this will provide some indication of the quality of the test set. The ratio of dead mutants to the total number of inequivalent mutants is the test data adequacy (TDA) of the test set. The same term is also used for the ratio of exposed to seeded faults with the $\mathrm{C}$ versions.

Test expressions were generated by OBJTEST, initially using the lowest operator nesting and minimum of parameter values. After the initial study an investigation was conducted to see how varying the number of values and other alternative techniques would affect the number of test expressions being produced and their fault exposing ability.

\section{RESULTS}

Faults were seeded into the $\mathrm{C}$ programs according to the results from the study done by Ostrand and Weyuker and details are given in Table 1. The size of the subject programs (LOC) and the number of mutants generated by Mothra and OBJTEST are given in Table 2 . 
Table 2 Size and number of faults inserted / mutants generated for subject programs

\begin{tabular}{lcrcccr}
\hline Subject & \multicolumn{2}{c}{ OBJ } & \multicolumn{2}{c}{ C } & \multicolumn{2}{c}{ Fortran } \\
\cline { 2 - 7 } & LOC & Mutants & LOC & Faults & LOC & Mutants \\
\hline Student Database & 159 & 99 & 171 & 27 & 263 & 866 \\
Airport & 264 & 135 & 489 & 51 & 594 & 5006 \\
\hline
\end{tabular}

Table 3 TDA of test sets generated from student database specification

\begin{tabular}{lrlll}
\hline Test Set & Size & OBJ TDA & C TDA & Fortran TDA \\
\hline $\mathrm{a}$ & 324 & 87.1 & 77.8 & 81.9 \\
$\mathrm{~b}$ & 2250 & 84.3 & 77.8 & 81.2 \\
$\mathrm{c}$ & 12402 & 92.9 & 81.5 & 87.6 \\
$\mathrm{~d}$ & 2250 & 70.0 & 81.5 & 85.5 \\
$\mathrm{e}$ & 13770 & 100.0 & 81.5 & 87.6 \\
$\mathrm{fa}$ & 36 & 46.5 & 22.2 & 24.5 \\
$\mathrm{fb}$ & 146 & 77.8 & 63.0 & 67.3 \\
$\mathrm{fc}$ & 146 & 78.8 & 66.7 & 70.2 \\
$\mathrm{fd}$ & 146 & 81.8 & 66.7 & 71.7 \\
$\mathrm{fe}$ & 290 & 81.8 & 66.7 & 71.7 \\
$\mathrm{ra}$ & 36 & 46.5 & 22.2 & 25.4 \\
$\mathrm{rb}$ & 146 & 70.7 & 63.0 & 61.5 \\
$\mathrm{rc}$ & 146 & 69.7 & 65.6 & 69.6 \\
$\mathrm{rd}$ & 146 & 72.7 & 66.7 & 70.7 \\
$\mathrm{re}$ & 290 & 75.8 & 66.7 & 71.7 \\
\hline
\end{tabular}

\subsection{Student database results}

The different test sets generated for the student database were:

- $a$ - operator nesting level of 2 , using the two names and two numbers.

- $b$ - operator nesting level of 3 , using two names and one number.

- $c$-operator nesting level of 3 , using one name and two numbers.

- $d$-operator nesting level of 3 , using two names, two numbers but expressions containing only one of the sex constants (male,female).

- $e$ - operator nesting of level 3 , using two names and two numbers.

The size of the test sets generated and the test data adequacy (TDA) for these test sets, $a$ to $e$, for the three versions, OBJ, C and Fortran, are given in Table 3.

For some of the test sets the number of test expressions being generated was very large indeed. Many of the expressions were considered similar. For example, because a set notation was being used to represent the student database and the operations on it, test expressions were produced containing Null and the set union operator, such as:

addmale ( Fred, 0, Null U Null ) 
Table 4 Size of test pools for the student database and airport scheduler specification

\begin{tabular}{lcr}
\hline Test category & \multicolumn{2}{c}{ Size } \\
& Student & Airport \\
\hline $\mathrm{a}$ & 10404 & 3180 \\
$\mathrm{~b}$ & 20350 & 1590 \\
$\mathrm{c}$ & 20350 & 1590 \\
$\mathrm{~d}$ & 20350 & 273 \\
$\mathrm{e}$ & 66854 & 684 \\
\hline
\end{tabular}

It was felt that expressions like this achieved very little beyond the equivalent expressions where union with Null (the identity for set union) is removed:

addmale ( Fred, 0, Null )

When analysing the output from Mothra it was discovered that many of the test expressions at the start of the test sets were killing large numbers of mutants and the remaining expressions were only killing a very small number each, if any at all. This led to the belief that choosing a small number of expressions from the total number of test expressions would provide comparable results. To investigate this various different strategies were tried. They were:

- Use only the first $5,10,15,20,25$ test expressions generated from the major operators: addmale, addfemale, deletemale, deletefemale, findmales and findmales. Because there are six major operators taking the first $5,10,15,20,25$ would produce test sets of size $30,60,90,120$ and 150 . These sets are labeled $a 5$ to $e 5, a 10$ to $e 10$ through to a25 to e25.

- Use only expressions that have an operator that does not alter the state of the database outermost; these are called evaluators and for the student database these were the operators findmales and findfemales, and an addmale or addfemale operator innermost. If two expressions are similar then remove one of them; also if the test set is generated to a nesting depth of $n$ then do not include expressions at depth less than $n$. Finally remove symmetrical expressions. These sets are labeled $f a$ to $f e$. The test sets generated using this strategy did not include test expressions generated using any other method.

The size and the test data adequacy of these test sets are given in Tables 3 and 5 . Random test data with evaluators outermost was generated to compare with the test sets $f a$ to $f e$. These sets $r a$ to $r e$ are also presented in Table 3.

The results of test sets $a 5$ to $e 5$ through to $a 25$ to $e 25$ were compared with random test data generated for each category, $a-e$. For each category, five names and five numbers were used and large test sets were generated. The same constraints were applied on these test sets as the initial test sets. The size of the test pools that expressions were chosen from are given in Table 4. Expressions were taken randomly from these sets in sizes comparable to the size of the original test sets. Results for the random test sets are given in Table 5 . 
Table 5 TDA of 'first 5, 10, 15, 20, 25' test expression sets generated from student database and random test sets

\begin{tabular}{lcccccc}
\hline Test Set & \multicolumn{2}{c}{ OBJ TDA } & \multicolumn{2}{c}{ C TDA } & \multicolumn{2}{c}{ Fortran TDA } \\
& Initial & Random & Initial & Random & Initial & Random \\
\hline a5 & 83.8 & 59.2 & 61.7 & 61.6 & 29.6 & 30.7 \\
b5 & 57.6 & 66.7 & 67.8 & 47.5 & 37.0 & 39.7 \\
c5 & 55.6 & 63.0 & 68.2 & 60.6 & 59.3 & 62.6 \\
d5 & 56.6 & 66.7 & 68.1 & 57.6 & 55.6 & 57.5 \\
e5 & 56.6 & 63.0 & 68.0 & 54.5 & 48.1 & 51.3 \\
a10 & 84.8 & 63.0 & 61.7 & 73.7 & 33.3 & 33.3 \\
b10 & 58.6 & 63.0 & 67.8 & 56.6 & 55.6 & 57.3 \\
c10 & 56.6 & 66.7 & 70.8 & 62.6 & 63.0 & 67.2 \\
d10 & 57.6 & 66.7 & 70.1 & 61.6 & 63.0 & 67.2 \\
e10 & 57.6 & 63.0 & 69.2 & 59.6 & 63.0 & 67.2 \\
a15 & 84.9 & 63.0 & 68.2 & 77.8 & 44.4 & 47.0 \\
b15 & 58.6 & 66.7 & 68.3 & 62.6 & 59.3 & 60.9 \\
c15 & 59.6 & 63.0 & 71.0 & 68.7 & 63.0 & 68.6 \\
d15 & 59.6 & 70.4 & 72.6 & 67.7 & 66.7 & 69.2 \\
e15 & 59.6 & 70.4 & 74.4 & 65.7 & 66.7 & 69.7 \\
a20 & 84.9 & 63.0 & 68.2 & 77.8 & 44.4 & 48.6 \\
b20 & 58.6 & 66.7 & 68.3 & 70.7 & 59.2 & 60.9 \\
c20 & 59.6 & 63.0 & 71.0 & 70.7 & 66.7 & 70.6 \\
d20 & 59.6 & 70.4 & 72.6 & 70.7 & 66.7 & 70.7 \\
e20 & 59.6 & 70.4 & 74.4 & 70.7 & 66.7 & 70.7 \\
a25 & 84.9 & 66.7 & 70.4 & 77.8 & 48.1 & 49.0 \\
b25 & 61.6 & 66.7 & 71.0 & 71.7 & 63.0 & 66.9 \\
c25 & 59.6 & 70.4 & 75.3 & 70.7 & 66.7 & 70.7 \\
d25 & 61.6 & 74.1 & 75.9 & 70.7 & 66.7 & 71.1 \\
e25 & 62.6 & 70.4 & 76.4 & 71.7 & 66.7 & 71.6 \\
\hline & & & & & & \\
\hline
\end{tabular}

Table 6 TDA of initial test sets for airport specification

\begin{tabular}{lrccc}
\hline Test Set & Size & OBJ TDA & C TDA & Fortran TDA \\
\hline $\mathrm{a}$ & 186 & 17.8 & 84.3 & 75.2 \\
$\mathrm{~b}$ & 93 & 14.8 & 52.9 & 35.4 \\
$\mathrm{c}$ & 93 & 17.0 & 62.7 & 75.1 \\
$\mathrm{~d}$ & 66 & 58.5 & 56.8 & 74.5 \\
$\mathrm{e}$ & 112 & 57.0 & 60.8 & 74.6 \\
$\mathrm{fe}$ & 1.02 & 91.1 & 72.5 & 67.7 \\
$\mathrm{te}$ & 1.12 & 84.4 & 72.5 & 81.5 \\
\hline
\end{tabular}


Table 7 TDA of random test sets for the airport scheduler specification

\begin{tabular}{llll}
\hline Test Set & OBJ TDA & C TDA & Fortran TDA \\
\hline a30 & 15.6 & 60.8 & 34.0 \\
b30 & 13.3 & 58.8 & 18.8 \\
c30 & 15.6 & 66.7 & 34.4 \\
d30 & 56.3 & 62.7 & 37.5 \\
e30 & 62.0 & 66.7 & 43.9 \\
a60 & 15.6 & 62.7 & 39.7 \\
b60 & 13.3 & 62.7 & 18.9 \\
c60 & 15.6 & 66.7 & 38.2 \\
d60 & 57.0 & 66.7 & 37.9 \\
e60 & 63.0 & 74.5 & 44.0 \\
a90 & 15.6 & 62.7 & 39.7 \\
b90 & 17.8 & 62.7 & 22.2 \\
c90 & 20.0 & 66.7 & 38.2 \\
d90 & 57.0 & 66.7 & 43.2 \\
e90 & 63.0 & 76.5 & 47.0 \\
a120 & 15.6 & 64.7 & 39.7 \\
b120 & 17.8 & 62.7 & 22.2 \\
c120 & 20.0 & 66.7 & 38.2 \\
d120 & 57.0 & 66.7 & 43.2 \\
e120 & 63.0 & 76.5 & 47.3 \\
a150 & 20.7 & 64.7 & 60.3 \\
b150 & 17.7 & 64.7 & 22.5 \\
c150 & 20.7 & 78.4 & 62.8 \\
d150 & 57.0 & 66.7 & 68.0 \\
e150 & 67.4 & 76.5 & 51.2 \\
\hline & & &
\end{tabular}

\subsection{Airport specification results}

The test sets generated from the airport specification were produced using the following constraints:

- a - Level 2 nesting of operators with evaluators outermost.

- b - Level 2 nesting of operators with evaluators outermost. Using the same constraints as for test set $a$ except for de-selection of the constant TODAY.

- $\mathrm{c}$ - Level 2 nesting as above but with just TODAY, i.e. not including the empty set.

- $\mathrm{d}$ - Level 1 nesting not just with the evaluators outermost and including TODAY and the empty set.

- e - Level 1 nesting as above but including two constants, one containing details of just one flight and the other containing details of two flights.

The size and the TDA of the described test sets are in Table 6 . 
Overall the results being achieved were reasonably satisfactory, but in an attempt to improve upon them additional test sets were generated. These were a test set with an extra flight number (fe) and a, test set with two extra invalid time values (te). The results for these test sets are given in Table 6 .

Random test sets were again used for comparison purposes. Test pools were produced for the major test sets by using extra flight, plane and time values and the constants containing flight details included or excluded accordingly. Sizes taken were 30, 60, 90, 120 and 150, with constraints as applied to sets $a, b, c, d$ and $e$. Given in Table 4 is the size of the test pool used. The results are presented in Table 7.

\section{CONCLUSION}

This paper has presented an investigation into the quality of test expressions generated from algebraic specifications, using two medium-sized specifications and their equivalent implementations in Fortran and C. Faults were inserted in the $\mathrm{C}$ versions using as far as possible the same types and proportions as found in a study of real industrial software. Mutation analysis was used on the Fortran versions and the OBJ specifications themselves. Assessed using this method, the fault detection ability of the initial test sets generated from the student database ranged from $70.0 \%$ to $100.0 \%$ for the OBJ, $77.8 \%$ to $81.5 \%$ for the $\mathrm{C}$ and $81.9 \%$ to $87.6 \%$ for the Fortran versions. For the airport specification the fault detection ability ranged from $14.8 \%$ to $91.15 \%$ for the OBJ, $52.9 \%$ to $72.5 \%$ for the $\mathrm{C}$ and $35.4 \%$ to $81.5 \%$ for the Fortran versions. In an attempt to generate some heuristic guidelines for producing a small number of test expressions that detect a large number of faults, different techniques and strategies were investigated. Although not as effective as the initial test sets, the number of test expressions generated are significantly smaller for only a slightly worse faul detection ability than the best case. The effectiveness of these simple strategies have been evaluated against random test sets of the same size. The random test sets performed slightly worse.

It is acknowledged that for experiments of this nature to provide concrete conclusions requires more and larger specifications together with equivalent implementations and that rigorous statistical methods be applied. The experimental work conducted by Hutchins et al (1991), on comparing the effectiveness of dataflow-based and controlflow-based test data adequacy, considered that the results obtained using seven subject programs that produced 130 faulty programs was severely limited. Obtaining moderate-sized specifications and equivalent implementations that have both been produced by independent sources is a limiting factor in this research. Using smaller specifications and implementations will not produce many faulty versions that can be used to assess effectively the quality of automatically generated test sets.

The tentative conclusion seems to be that test expressions from algebraic specifications can be effective at exposing faults in implementations. Although different specifications will probably need to be treated on an individual basis, depending on the abstract data type being used to implement the operations. This is because the number of test expressions that could be generated can become extremely large and many of them may detect very few additional faults. There does not appear to be an obvious relationship between the number of specification mutants killed and the number of faults exposed in the $\mathrm{C}$ implementation or the number of mutants killed in the Fortran implementation. The results 
show that at deeper levels of nesting more faults are exposed. This was also suggested by Doong and Frankl (1991). It could be that the more operations that are applied to a data structure, the more likely it is that a fault will produce erroneous data values that are amplified by the long sequence of operations. It is also possible that many different operators appearing in a test expression will cover a large part of the implementation's functionality and thereby expose many faults. It was discovered that for a test expression to be able to kill a mutant of the specification, the expression has to be nested to at least the same depth as the operator nesting in the equation under test. The majority of equations describing the operators of the airport specification were at depth 3 . This explains why the first three test sets did not kill many specification mutants at level 2 nesting. The effective depth of the remaining test sets was at least 3 or 4 .

The authors look forward to building upon these experiments. Perhaps by including additional techniques that will allow better analysis of possible parameter values or by determining the quality of the formal specification, the number of faults detected can be increased and the number of test expressions can be decreased. In particular, the authors are considering ways of reducing the number of test expressions automatically generated by exploiting symmetry and other techniques.

\section{REFERENCES}

Bernot, G., Gaudel, M., and Marre, B. (1991), Software testing based on formal specifications: a theory and a tool. Software Engineering Journal, 6(6):387-405.

Bougé, L., Choquet, N., Fribourg, L., and Gaudel, M. (1986), Test sets generation from algebraic specifications using logic programming. The Journal of Systems and Software, 6(4):343-360.

Coleman, D., Gallimore, R., and Stavridou, V. (1987), The design of a rewrite rule interpreter from algebraic specifications. Software Engineering Journal, 2(4):95-104.

Doong, R. and Frankl, P. (1991), Case studies on testing object-oriented programs. In Proceedings of the Symposium on Testing, Analysis and Verification (TAV4), pages 165-177, Victoria, B.C., Canada. ACM Press.

Gannon, J., McMullin, P., and Hamlet, R. (1981), Data-abstraction implementation, specification and testing. ACM Transactions on Programming Language and Systems, 3(3):211-223.

Gerrard, C., Coleman, D., and Gallimore, R. (1990), Formal specification and design time testing. IEEE Transactions on Software Engineering, 16(1):1-12.

Goguen, J. A. and Tardo, J. J. (1979), An introduction to Obj. In Proceedings of Specifications of Reliable Software, pages 170-189. IEEE Computer Society Press.

Hall, P. (1988), Testing with respect to formal specification. In Proceedings of the Second IEE/BCS Conference 'Software Engineering 88', pages 159-163. University of Liverpool, IEE/BCS.

Hutchins, M., Foster, H., Goradia, T. and Ostrand, T. (1994), Experiments on the effect iveness of dataflow- and controlflow-based test adequacy criteria. In Proceedings of the 16th International Conference on Software Engineering, pages 191-200. IEEE Computer Society Press.

Jalote, P. (1989), Testing the completeness of specifications. IEEE Transactions on Software Engineering, 15(5):526-531. 
King, K. N. and Offutt, A. J. (1991), A Fortran language system for mutation-based software testing. Software-Practice and Experience, 21(7):685-718.

Offutt, A. J. and Craft, W. M. (1994), Using compiler optimization techniques to detect equivalent mutants. Software Testing, Verification and Reliability, 4(3):131-154.

Ostrand, T. and Weyuker, E. (1984), Collecting and categorizing software error data in an industrial environment. The Journal of Systems and Software, 4(4):289-300.

Rolland, F. (1992), Programming with VDM, chapter 5, pages 42-52. MacMillan.

Stocks, P. and Carrington, D. (1993), Test template framework: A specification-based testing case study. In Proceedings of the 1993 International Symposium on Software Testing and Analysis (ISSTA 1993), pages 11-18. ACM Press.

Woodward, M. (1993), Errors in algebraic specifications and an experimental mutation testing tool. Software Engineering Journal, 8(4):211-224.

\section{BIOGRAPHY}

Stephen P. Allen received a BSc ( Hons) degree in computer science in 1993 from the University of Liverpool and is currently working towards a $\mathrm{PhD}$ at the same institution.

His current research interests include software testing and software specification.

Martin R. Woodward received a BSc ( Hons ) degree in mathematics in 1969 and a PhD degree in applied mathematics in 1972, both from the University of Nottingham in the UK.

For three years he was employed by the University of Oxford as a Research Assistant on secondment to the UK Atomic Energy Authority at the Culham Laboratory. He has been at the University of Liverpool since 1975 where he is now a Senior Lecturer in the Computer Science Department. His current research interests include: software specification, software testing and analysis, software metrics, and software development environments.

Dr. Woodward is Editor of the journal Software Testing, Verification and Reliability ( Wiley ) and is also on the Editorial Board of the Software Quality Journal ( Chapman and Hall ). He has also served on the Programme Committees of several international conferences, including recently the International Symposium on Software Testing and Analysis (ISSTA-96) held in San Diego, USA, in January 1996. 